ARTICLE

\title{
Application and Validation of Particle Transport Code PHITS in Design of J-PARC 1 MW Spallation Neutron Source
}

\author{
Masahide HARADA*, Fujio MAEKAWA, Kenichi Oikawa, Shin-ichiro MEIGO, \\ Hiroshi TAKADA and Masatoshi FUTAKAWA
}

Japan Atomic Energy Agency, 2-4 Shirakata, Tokai-mura, Naka-gun, Ibaraki-ken, 319-1195, Japan

\begin{abstract}
The Japan Spallation Neutron Source (JSNS) is one of the major experimental facilities in the Japan Proton Accelerator Research Complex (J-PARC). JSNS operates with $3 \mathrm{GeV}$ and $1 \mathrm{MW}$ pulsed proton beams, and has the highest-class neutron intensity in the world. In the design stage, aiming for the best neutronic performance, the PHITS code was fully applied in the JSNS neutronics design, and calculations for several thousand cases were performed using complicated models. Not only optimization of neutronic performance, but also shielding calculation, nuclear heat estimation for the engineering design, residual radioactivity estimation for the cask design, and radiation damage estimation for the life and maintenance design were also done with the PHITS code. JSNS is one of the first facilities in the world to fully adapt such a simulation code to the neutronics design. In these calculations, note the change in particle energy from $\mathrm{GeV}$ to $\mathrm{meV}$ (12 decades), and that neutron fluxes are reduced by 10 decades or more. To confirm their reliability, these calculations were validated by measurement data for neutron spectral intensities, pulse shape, nuclear heat generation, and radioactivity. The results showed that the calculated values were in good agreement with the measured values. We confirmed that the PHITS code was reliable for such design calculations. At the same time, this indicates that the PHITS code is a powerful tool for neutronic design in other radiation source facilities and accelerator facilities.
\end{abstract}

KEYWORDS: PHITS, NMTC/JAM, JSNS, J-PARC, spallation neutron source, validation

\section{Introduction}

The Japan Proton Accelerator Research Complex (J-PARC) has been constructed in Tokai-mura, Ibaraki-ken, Japan. The Materials and Life Science Experimental Facility (MLF) is one of the major experimental facilities in J-PARC, and the Japan Spallation Neutron Source (JSNS) is located in the MLF. JSNS produced its first neutron beam on May $30,2008,{ }^{1)}$ and the user program at JSNS commenced in December 2008. A mercury (Hg) target is bombarded by a pulsed proton beam to produce spallation neutrons, with a repetition rate of $25 \mathrm{~Hz}$. As of June 2010, the proton beam power for regular operation was $120 \mathrm{~kW}$, and will eventually be increased to $1 \mathrm{MW}$. The neutrons are slowed down by three supercritical hydrogen $\left(\mathrm{H}_{2}\right)$ moderators to produce cold neutrons, and neutron beams are delivered to user's neutron scattering instruments through a total of 23 beam ports (BLs) Figure 1 shows the typical neutron flux in a spallation neutron source compared with a light water reactor and a fast breeder reactor.

In the design stage of JSNS there were many design parameters to determine in optimization studies to pursue better neutronic performance. Owing to the recent remarkable progress in computing technology and the rapid drop in the price of personal computers, these design parameters were derived by neutronic simulation calculations. However, at the time of the design stage, there was no such code that

*Corresponding author, E-mail:harada.masahide@jaea.go.jp could be used for the JSNS design, because we needed to deal with particle transport phenomena in a wide energy range beyond $20 \mathrm{MeV}$ up to $3 \mathrm{GeV}$. To meet this need, the NMTC/JAERI code was upgraded by improving the precision of its high-energy physics models and several user interfaces. ${ }^{2-4)}$ The NMTC/JAERI code has been validated in several benchmark experiments to check its reliability. ${ }^{5,6}$ The NMTC/JAERI code was then further improved to become the NMTC/JAM code and finally evolved into the Monte Carlo particle and heavy ion transport code PHITS. ${ }^{7-9)}$ The PHITS code can deal with particle transport phenomena in a wide energy range from $\mu \mathrm{eV}$ up to $\mathrm{TeV}$.

The PHITS code was fully applied to the JSNS neutronic designs. Not only optimization of neutronic performance but also shielding design, nuclear heat estimation, residual radioactivity estimation, and radiation damage estimation of materials were carried out. This full application of such a neutronic calculation code to the design of a large, high-energy accelerator facility was one of the first such trials in the world. In order to calculate many cases with a large-sized model $(>15 \mathrm{~m})$, a large number of models $(>2,000)$ and a large neutron flux attenuation (>10 decades) in a realistic computation time, a parallel computing system was prepared consisting of 36 commercially available personal computers (Pentium IV, dual core, $2.8 \mathrm{GHz}$ ) running free software.

Since JSNS started operation, a large amount of commissioning data has been obtained. Neutron spectral intensity and pulse shape at each beam port are the most important

(C) 2011 Atomic Energy Society of Japan, All Rights Reserved. 


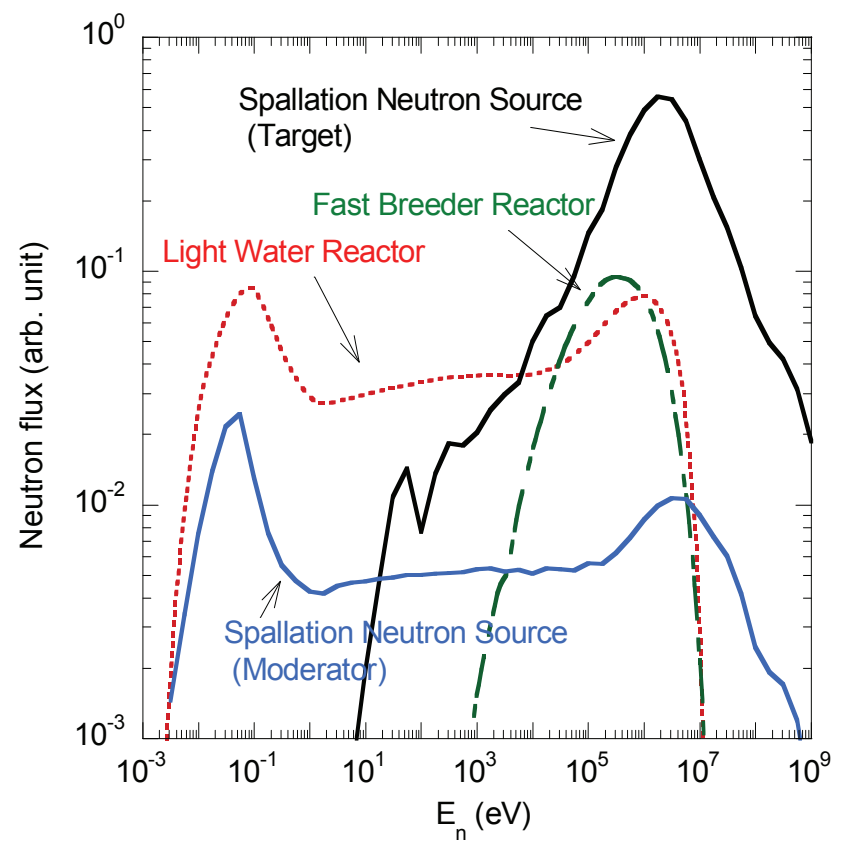

Fig. 1 Typical calculated neutron flux in spallation neutron source, light water reactor and fast breeder reactor

data, because they are directly used in designing user's instruments, which are optimized to create leading-edge scientific outcomes. The nuclear heat generation data are also important in such a high intensity accelerator facility because they are required for heat removal design, structural design, and cooling system design. Evaluation of radioactive inventory is also required for cask design and estimation of exposure doses to rad-workers, and so on. High accuracy in these calculated nuclear properties is needed in terms of safety, neutronic performance, construction cost saving, and so on. In this paper, several important calculated data sets are compared with the corresponding measured data: neutron spectral intensity, pulse shape, nuclear heat generation and radioactivity, and reliability of the design calculations will be discussed.

\section{Calculation Method}

\section{Design Code System}

The design code system prepared for JSNS is shown in Fig. 2. The main code in this system is the PHITS code with the JENDL-3 evaluated cross section library. ${ }^{10,11)}$ Proton beam profiles were first simulated by the Decay-Turtle code, ${ }^{12)}$ and the output was used as input data for the PHITS calculations. Protons, neutrons, photons, electrons, and other minor particles are tracked by PHITS code with detailed models. Data needed to evaluate the lifetime of components due to radiation damage of materials, such as DPA (displacement per atom), and hydrogen and helium yield, are calculated directly with the PHITS code. As shown in Fig. 2, the output of the PHITS code is either directly used data or is processed by other codes (DCHIAN-SP, ${ }^{13)}$ MCNPX, ${ }^{14}$ $\mathrm{MCNP},{ }^{15)}$ and so on) to obtain data for mechanical engineering design, shielding design, and so on.

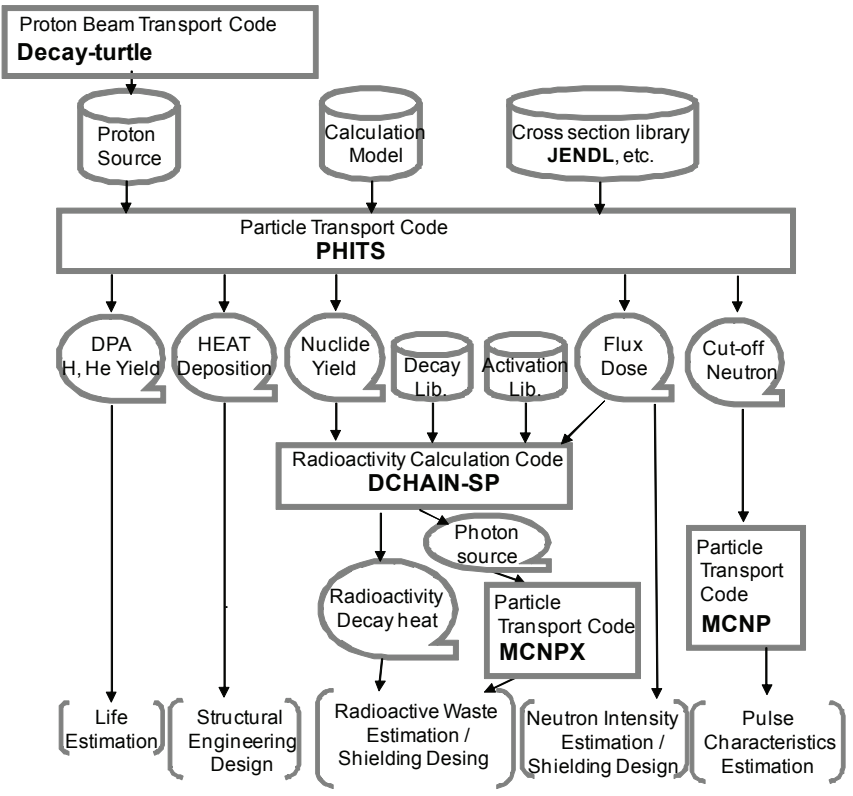

Fig. 2 Design code system for JSNS

\section{Outline of JSNS and Calculation Model}

JSNS consists of a proton beam line, target station, and neutron beam lines with shields. In the target station, the $\mathrm{Hg}$ target is surrounded by Beryllium (Be) and Iron $(\mathrm{Fe})$ reflectors. The three $\mathrm{H}_{2}$ moderators, which comprise a coupled moderator (CM), decoupled moderator (DM) and decoupled poisoned moderator (PM), are located above and below the target. Because each moderator provides neutron pulses with different characteristics, a moderator is selected to conform to neutron scattering experiments. Super-critical hydrogen is used as a moderator material, which maintains nearly $100 \%$ para-hydrogen states. The 23 BLs equipped downstream of the source are located in two experimental halls with beam line devices. At each beam line, neutron beams are extracted from the CM, DM, or PM. The CM, DM, and PM have 11, 6, and 6 BLs, respectively. Figure 3 shows the arrangement of the neutron beam lines at JSNS in March 2010. ${ }^{16)}$

Precise calculation models are indispensable for precise calculations. A detailed calculation model of JSNS (engineering calculation model) is shown in Fig. 4. In this model, realistic shapes, thicknesses, positions, and materials of the $\mathrm{Hg}$ target, the $\mathrm{Be}-\mathrm{Fe}$ reflectors, and the three $\mathrm{H}_{2}$ moderators were considered. Multiple layers of the target and the moderator, the cooling channels in the reflector and the neutron absorbing materials were also considered as precisely as possible. The kernel data estimated by MacFarlane, et al. was used to precisely treat neutron scattering in thermal and cold neutron regions in the $\mathrm{H}_{2}$ moderators. ${ }^{17)}$ Water-cooled or air-cooled shielding blocks were assumed as homogeneous materials on the outside of the reflectors. Major parameters of the engineering calculation model and calculation conditions are summarized in Table $\mathbf{1 .}$

\section{Calculation of Neutron Spectral Intensities and Pulse Shape at Each Beam Line}

For the calculation of neutron spectral intensities at lower 


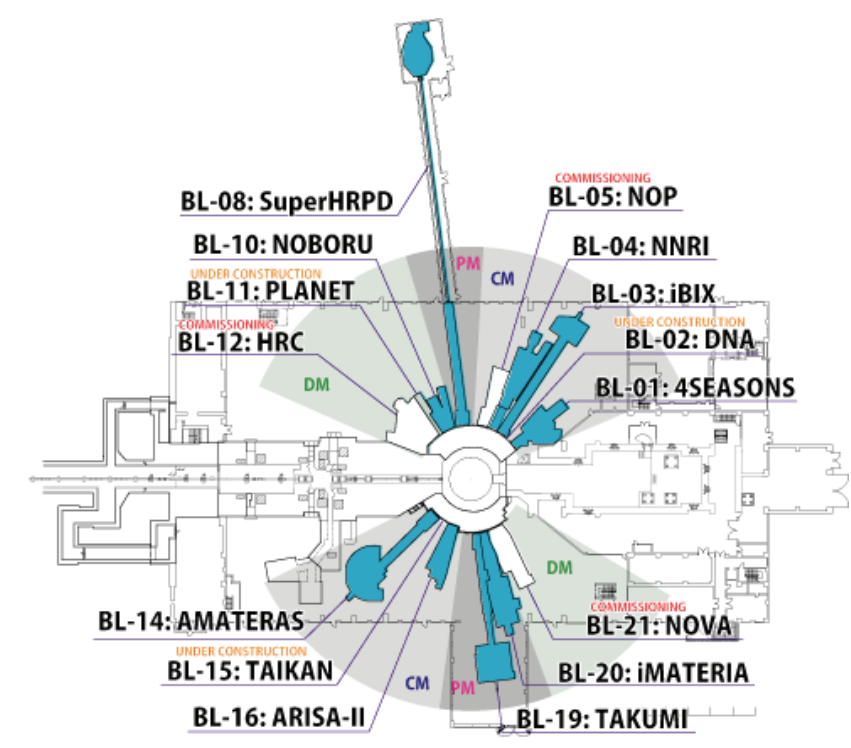

Fig. 3 Arrangement of neutron beam lines at JSNS in Mar. 2010

energy (below $1 \mathrm{MeV}$ ), a point estimator function was used in MCNP. MCNP was used because the point estimator function is not available in the PHITS code. This estimator was located on the beam axis at $10 \mathrm{~m}$ from the moderator. However, due to the absence of cross section data in JENDL, the point estimator function was not available in the higher energy region (above $20 \mathrm{MeV}$ ). Therefore, first, the energy-dependent angular distribution of leakage neutrons from the moderator and the reflector towards downstream of the beam were calculated. The neutron spectral intensity data along the neutron beam line to reach the sample position at each beam line were then calculated from the angular distribution data. These data were applied in the energy region above $1 \mathrm{MeV}$.

For the pulse shape calculations, the point estimator function was also used. The point estimator was also located $10 \mathrm{~m}$ from the moderator. Though particle flight times are counted in the estimator, the flight time between the moderator and the estimator was eliminated to reduce variance.

Data for neutron spectral intensities and pulse shapes at each beam line have been made available on the J-PARC website. $^{16)}$

\section{Calculation of Nuclear Heat Generation}

The maximum cooling ability of a circulation system is assumed from the heat load data. Over-estimation of the heat load therefore pushes up the cost of the circulation system, and especially the cryogenic circulation system. Precise nuclear heat data are thus necessary to minimize costs. In order to obtain precise heat disposition data, electron transports and revised kerma factors were considered. The kerma factor refers to kinetic energy released in matter per particle. All particle transports were also considered.

Electron transport was used to treat the nuclear heat generation of photons precisely. Photon kerma factors are sometimes used for calculation of nuclear heating due to photons. This calculation procedure relies on one point ap-

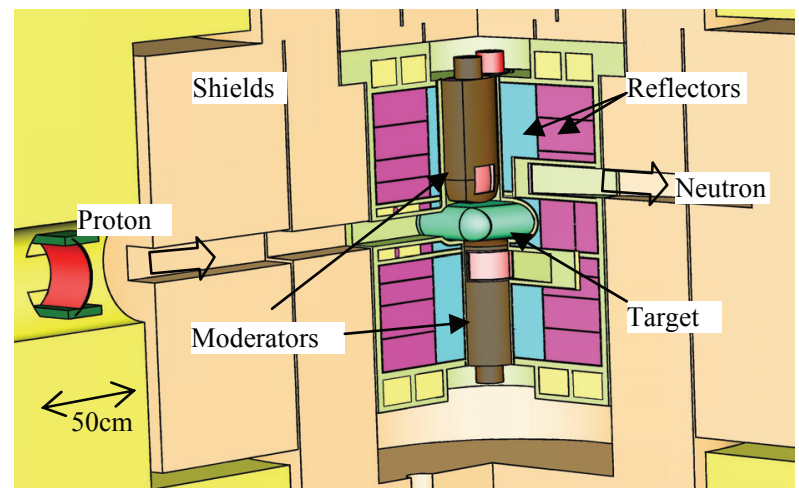

Fig. 4 JSNS calculation model

Table 1 Calculation condition

\begin{tabular}{|c|c|}
\hline Item & Calculation condition \\
\hline \multicolumn{2}{|l|}{ Proton Beam } \\
\hline Power & $1 \mathrm{MW}$ at the proton beam window \\
\hline Operation time & 5000 hours / year \\
\hline \multirow{3}{*}{ Profile } & Emittance : $81 \pi \mathrm{mm} \mathrm{mrad}$ \\
\hline & Gaussian + Uniform \\
\hline & Footprint : $180 \times 70 \mathrm{~mm}^{2}$ \\
\hline Repetation rate & $25 \mathrm{~Hz}$ \\
\hline \multicolumn{2}{|l|}{ Proton Beam window } \\
\hline Material \& thickness & Al-alloy (A5083), $2.5 \mathrm{~mm}^{\mathrm{t}} \times 2$ plates \\
\hline Coolant & $\mathrm{H}_{2} \mathrm{O}$ \\
\hline \multicolumn{2}{|l|}{ Target } \\
\hline Material, density & Mercury, $13.6 \mathrm{~g} / \mathrm{cm}^{3}$ \\
\hline Vessel material & 316L stainless steel \\
\hline Coolant & $\mathrm{D}_{2} \mathrm{O}$ \\
\hline \multicolumn{2}{|l|}{ Moderator } \\
\hline Type \& number & $\begin{array}{ll}\text { Coupled }(\mathrm{CM}) & 1 \\
\text { Decoupled (DM) } & 1 \\
\text { Decoupled Poisoned (PM) } & 1\end{array}$ \\
\hline Material property & $\begin{array}{l}\text { Super-critical hydrogen, } 20 \mathrm{~K} \text {, } \\
1.5 \mathrm{MPa}, 0.07 \mathrm{~g} / \mathrm{cm}^{3}\end{array}$ \\
\hline Vessel material & Al-alloy (A6061, A5083) \\
\hline Coolant & $\mathrm{H}_{2} \mathrm{O}$ \\
\hline \multicolumn{2}{|l|}{ Reflector } \\
\hline Material \& size (Inner) & Beryllium, $50 \mathrm{~cm}$ (Dia.) x $100 \mathrm{~cm}$ (Hei.) \\
\hline Material \& size (Outer) & Iron, $100 \mathrm{~cm}$ (Dia.) x $100 \mathrm{~cm}$ (Hei.) \\
\hline Coolant material., fraction & $\mathrm{D}_{2} \mathrm{O}$, about $10 \%$ (channel width: $5 \mathrm{~mm}$ ) \\
\hline Vessel material & Al-alloy (A5083) \\
\hline \multicolumn{2}{|l|}{ Water-cooled shield } \\
\hline Material & 304 stainless steel \\
\hline \multicolumn{2}{|c|}{ Coolant material, fractiol $\mathrm{H}_{2} \mathrm{O}$, about $10 \%$} \\
\hline \multicolumn{2}{|c|}{\begin{tabular}{|l|l} 
Air-cooled shield & \\
\end{tabular}} \\
\hline Material & Steel \\
\hline Coolant material & Air \\
\hline \multicolumn{2}{|l|}{ Neutron beam line } \\
\hline Number of beam lines & 23 \\
\hline
\end{tabular}

proximation in which the entire electron energy is deposited at the place where the electron is produced. This approximation can introduce meaningful inaccuracies into the calculated results when the flight path of an electron scattered by a photon is longer than a region size of interest.

Because some neutron kerma factors produced from evaluated nuclear data files were inconsistent in terms of the energy balance, revised kerma factors were used. Such inconsistency occurs when the original nuclear data files violate the energy balance, and therefore we adopted energy-consistent kerma factors for major materials $\left({ }^{27} \mathrm{Al}\right.$, ${ }^{\text {nat }} \mathrm{Fe}$, 


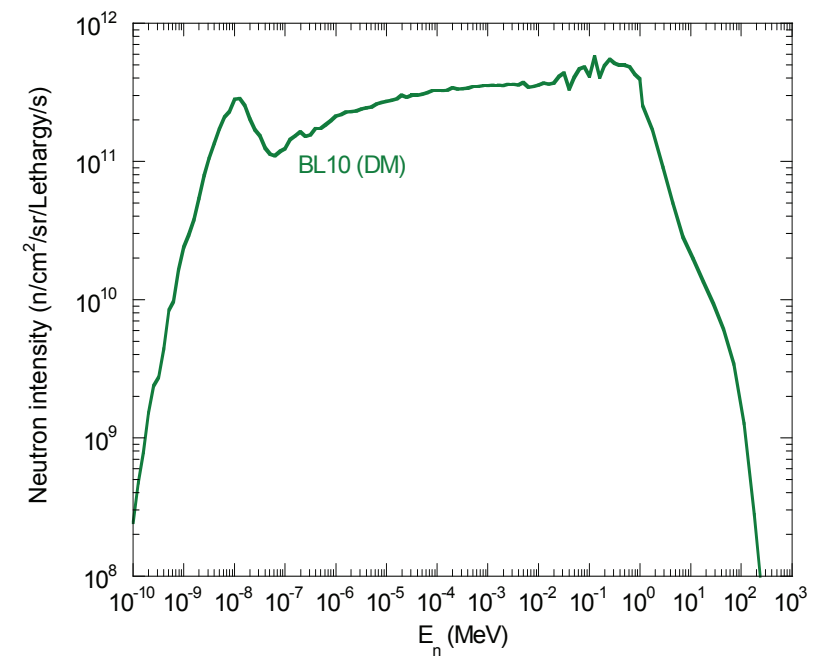

Fig. 5 Calculated neutron spectral intensities in the full energy region from $\mathrm{GeV}$ to $\mu \mathrm{eV}$ at $\mathrm{BL} 10$

and so on) in the energy region from about $1 \mathrm{MeV}$ to $20 \mathrm{MeV}$.

By taking such considerations into account, the total nuclear heat generation and its distribution were calculated for each component. Details of this calculation have also been reported. ${ }^{18)}$ These detailed procedures contributed to increasing the precision of the calculation up to $20 \%$.

\section{Calculation of Target Vessel Radioactivity}

The radioactivity of each component was calculated with the PHITS code and the DCHIAN-SP code. Firstly, neutron flux below $20 \mathrm{MeV}$ and nuclide yields from the nuclear reaction above $20 \mathrm{MeV}$ were calculated with the PHITS code. Then, from these data, radioactive nuclides and their decays were calculated with the DCHAIN-SP code. Next, decay gamma-ray transport calculations from residual radioactive nuclei were executed for estimation of dose rate and decay heat. These data were used for the cask design and planning of maintenance.

\section{Calculation Results}

\section{Neutron Spectral Intensities}

Figure 5 shows neutron spectral intensities at one of the BLs (BL10). Source intensity is defined for comparison of neutron source intensity, and is neutron intensity normalized by area, solid angle, energy, and time. These data were converted to source intensity from the view of the moderator surface and the distance from the moderator. A peak in the low-energy region is observed at $10 \mathrm{meV}$, although it is not a Maxwellian peak formed by thermalized neutrons. Neutron intensities in the energy region from $10 \mathrm{keV}$ to $100 \mathrm{meV}$ are slightly lower, which means that neutron leakage and/or absorption readily occur in this field. Maximum intensity is observed in the energy region around $1 \mathrm{MeV}$. The high-energy end of the spectrum is about $200 \mathrm{MeV}$. In the cold and thermal neutron region below $100 \mathrm{meV}$, neutron intensities depended on the kind of moderator (CM, DM, and $\mathrm{PM}$ ). On the other hand, in the high-energy region above

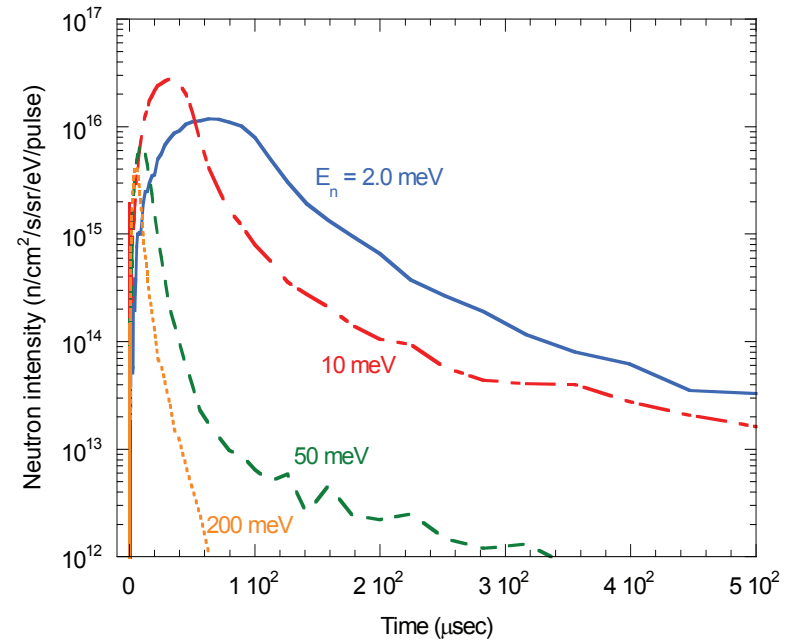

Fig. 6 Calculated pulse shape at $E_{n}=2,1050$ and $200 \mathrm{meV}$ at BL10

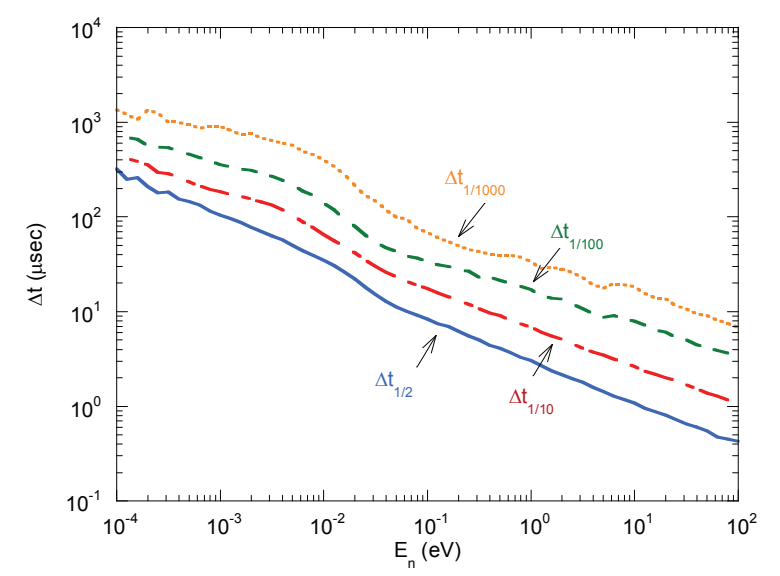

Fig. 7 Calculated pulse widths at half, 1/10, 1/100 and 1/1,000 maximum at BL10

$1 \mathrm{MeV}$, the intensities depend on the angle of the proton beam direction. That is, the neutron intensity in the high-energy neutrons becomes higher in a more forward beam line. These data were used as the source data for neutron beam line design and for neutron beam line shielding.

\section{Pulse Shape and Pulse Width}

Figure 6 shows pulse shapes at $\mathrm{En}=2,10,50$, and $200 \mathrm{meV}$ in BL10. In the energy region below $20 \mathrm{meV}$, certain pulse tails caused by thermalized neutrons in the moderator are observed from $1 / 10$ to approximately $1 / 100$ of the peak. Above $20 \mathrm{meV}$, as neutron energy increases, the pulse tail becomes smaller. Pulse widths at half, $1 / 10,1 / 100$, and 1/1,000 maximum are shown in Fig. 7. All pulse widths decrease as neutron energy increases, roughly in direct proportion to velocity. However, an increase in the pulse width in the energy region below $20 \mathrm{meV}$ is observed due to an increase in thermalized neutrons in the moderator.

\section{Nuclear Heat Generation}

Table 2 shows the calculated total nuclear heat generation values of major components in the case of the $1 \mathrm{MW}$ proton beam. The designs of the cooling circulation systems at each 
Table 2 Nuclear heat generation

\begin{tabular}{|l|l|c|}
\hline \multicolumn{2}{|l|}{ Component } & $\begin{array}{l}\text { Nuclear } \\
\text { Heating } \\
(\mathrm{kW})\end{array}$ \\
\hline \hline Target & Mercury \& Vessel & 493.0 \\
\hline Reflector & Reflector \& Vessel & 193.9 \\
\hline Shielding block & $\begin{array}{l}\text { Water-cooled } \\
\text { shielding block }\end{array}$ & 133.8 \\
\hline $\begin{array}{l}\text { Proton beam } \\
\text { window }\end{array}$ & Window assembly & 1.4 \\
\hline $\begin{array}{l}\text { Poisoned } \\
\text { moderator }\end{array}$ & $\mathrm{H}_{2}$ \& $\mathrm{H}_{2}$ Vessel & 1.1 \\
\cline { 2 - 3 } & $\begin{array}{l}\text { Water-cooled outer } \\
\text { Vessel }\end{array}$ & 4.4 \\
\hline $\begin{array}{l}\text { Decoupled } \\
\text { modearator }\end{array}$ & $\mathrm{H}_{2}$ \& $\mathrm{H}_{2}$ Vessel & 1.0 \\
\cline { 2 - 3 } & $\begin{array}{l}\text { Water-cooled outer } \\
\text { Vessel }\end{array}$ & 4.9 \\
\hline $\begin{array}{l}\text { Coupled } \\
\text { modearator }\end{array}$ & $\mathrm{H}_{2}$ \& $\mathrm{H}_{2}$ Vessel & 1.4 \\
\cline { 2 - 3 } & $\begin{array}{l}\text { Water-cooled outer } \\
\text { Vessel }\end{array}$ & 5.2 \\
\hline Total & & 874.7 \\
\hline
\end{tabular}

component were based on these data. The heat generation in the target is highest of all components, and accounts for about $50 \%$ of the heat generated by all components. Prompt nuclear heat generation in the hydrogen circulation loop was $3.5 \mathrm{~kW}$, as shown in Table 2 . An additional $0.2 \mathrm{~kW}$ decay heat was also estimated to be caused to the hydrogen circulation system due to its not negligible value for the system, resulting in a total heat load to the hydrogen circulation loop of $3.7 \mathrm{~kW}$. The total nuclear heat of all components $(875 \mathrm{kw})$ is smaller than the incident proton beam power (1 MW). This difference can be accounted for by considering escape particles and nuclear conversion. Firstly, because the detailed calculation model is a finite volume, some particles (neutrons and photons) escape from the model. The total kinetic energy of escaping particles is calculated as $16 \mathrm{~kW}$. Secondly, with regard to nuclear conversion, according to the PHITS code's output, the total increase in nuclear mass energy corresponds to $111 \mathrm{~kW}$. The sum of three values, $1,001 \mathrm{~kW}$, is very close to the incident proton beam power of $1 \mathrm{MW}$. This fact indicates the consistency of the nuclear heating calculation code system in terms of energy conservation.

\section{Comparison with Measurements}

\section{Neutron Spectral Intensities at BL10}

Figure 8 shows measured compared with calculated neutron spectral intensities at BL10. The measured data were obtained by the CTOF method and a detector with a half-inch high pressure He-3 detector. ${ }^{19,20)}$ The measured data were converted to source intensity for comparison with the calculated data. The calculated and measured values are in good agreement (within 20\%). Although the particle energy was changed from $\mathrm{GeV}$ to $\mathrm{meV}$ (12 decades) and neutron fluxes reduced by 10 decades or more, this is a noteworthy agreement.

\section{Neutron Spectral Intensities at Other Beam Lines}

Figure 9 shows measured compared with calculated neutron spectral intensities at BL04 and BL19. ${ }^{20)}$ In these measurements, the detector was located at the sample posi-

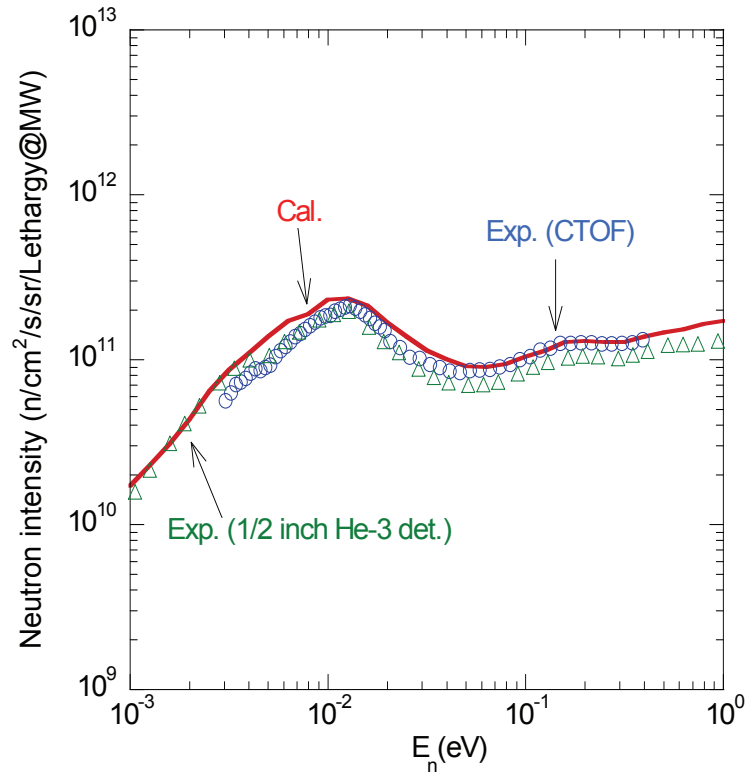

Fig. 8 Comparison of measured neutron spectral source intensities with calculated one at BL 10
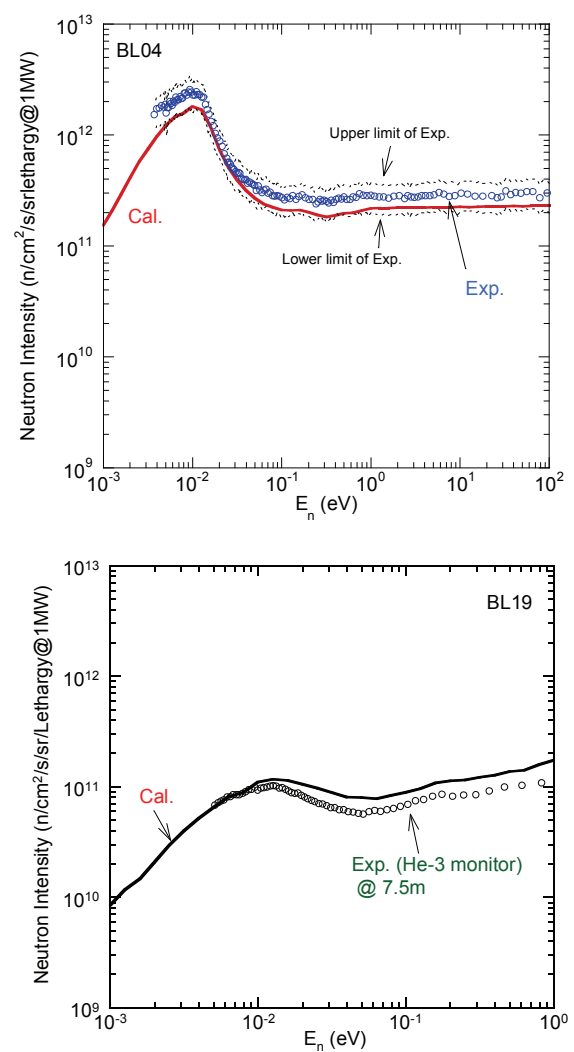

Fig. 9 Comparison of measured neutron spectral source intensities with calculated one at BL 04 and BL19. Experimental errors in the measurement at BL04 and BL19 are $30 \%$ and $10 \%$, respectively

tion. The experimental errors at BL04 and BL19 are considered to be within about $30 \%$ and $10 \%$, respectively, due to the differences in the measurement systems. The calculated and measured values at both BL04 and BL19 are in good agreement within the margin of error. 


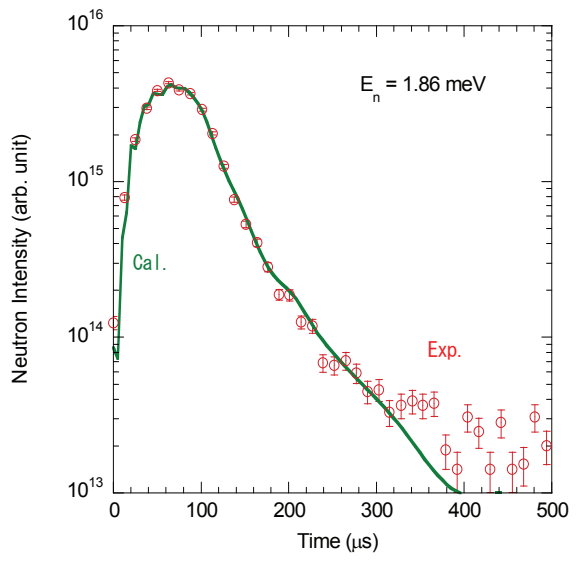

Fig. 10 Comparison of calculated pulse shape with measured ones at neutron energy of $1.8 \mathrm{meV}$

\section{Pulse Characteristics at BL10}

Simulated data were compared with the measured data. Bragg reflection of single-crystal mica was used for the pulse measurement. Mica at room temperature was placed at the sample position (14 $\mathrm{m}$ from the moderator) in BL10 and a He-3 detector ( 7 atoms) was placed behind $\left(170^{\circ}\right)$ the neutron beam direction. Figure 10 shows the pulse shape at $E_{n}=$ $1.8 \mathrm{meV}$. The measured and calculated values from the pulse peak to $1 / 100$ of the pulse peak are in good agreement. Figure 11 shows the measured pulse widths at half and $1 / 10$ (points) maximum compared with the calculated values (lines). The calculated and measured values are in good agreement in all energy regions.

\section{Nuclear Heat Generation at Target and Moderator}

At a commissioning operation (beam power: $285 \mathrm{~kW}$ ), the temperature of the $\mathrm{Hg}$ in the target rose up $8.9 \mathrm{~K}$ at the $\mathrm{Hg}$ flow of $31.5 \mathrm{~m}^{3} / \mathrm{h}$. From the simulated value as shown in Table 2, the calculated rise in temperature was $9.0 \mathrm{~K}$ at the same beam power. Therefore, the rise in temperature is in good agreement between the measured and simulated values.

At a commissioning operation (beam power: $307 \mathrm{~kW}$ ), the temperature of the hydrogen in the moderator rose up about $0.7 \mathrm{~K}$ to $1.0 \mathrm{~K}$ in three moderators with a flow rate of about $60(\mathrm{~g} / \mathrm{s})$ and a hydrogen temperature of $18 \mathrm{~K}$. The calculated rise in temperature was $0.9 \mathrm{~K}$ at the same beam power. ${ }^{21)}$ There is good agreement between the measured and calculated values, although the nuclear heat load to the hydrogen circulation system is only about $1 / 250$ of the incident proton beam power, which could result in large error being caused. The detailed procedures to increase the precision of the calculation noted above contributed to this agreement.

\section{Radioactivity of Target and Shutter}

After a commissioning operation (beam power: $4 \mathrm{~kW}$ ), the radioactivity of a target vessel made of stainless steel 316L was measured. The target hit by $3 \mathrm{GeV}$ proton beam was exposed to intense secondary protons and neutrons in all energy regions. Measured dose rates on the surface of the target head were 1 and $0.5 \mathrm{mSv} / \mathrm{hr}$ after 2 days cooling and 10 day cooling, respectively. The calculated values for the

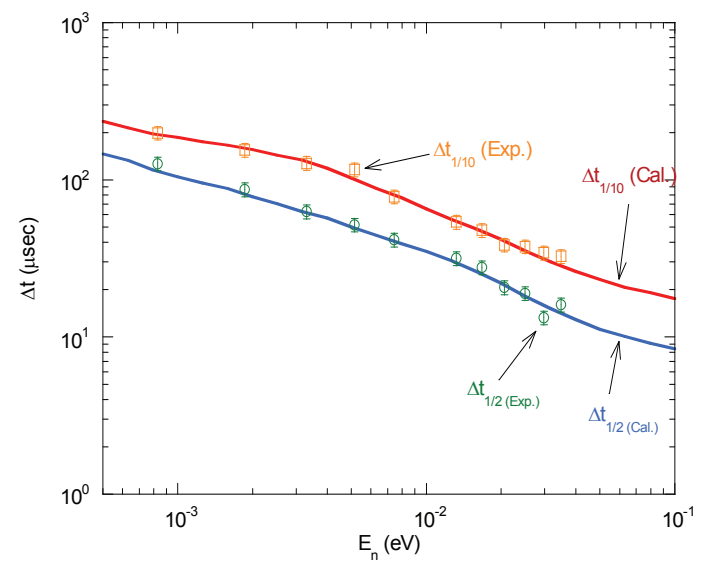

Fig. 11 Comparison of calculated pulse shape with measured ones at neutron energy of $1.8 \mathrm{meV}$

same conditions were 0.5 and $0.2 \mathrm{mSv} / \mathrm{hr}$, respectively. The calculated and measured values for the same conditions are in good agreement, within a factor of 3.

The calculated maximum dose rate with the shutter kept closed during the operation was $0.4 \mu \mathrm{Sv} / \mathrm{hr}$. The measured dose rate was $0.7 \mu \mathrm{Sv} / \mathrm{hr}$. This was a position where neutrons constantly hit a closed shutter position. The calculated and measured values are in good agreement, within a factor of 2.

\section{Conclusion}

Neutronic simulations were fully utilized for the design of JSNS, for the first time in the world for such a facility. The neutronic calculations for the JSNS design, such as the neutron intensity, the pulse shape, the nuclear heat generation, and the radioactivity, were in good agreement with the measured values. This result indicates that the neutronic design of JSNS is sufficiently reliable, and that the PHITS code system can be used for the neutronic design of other accelerators or nuclear radiation source facilities in energy ranges up to $\mathrm{GeV}$.

\section{Acknowledgment}

The authors would like to acknowledge the staff of the Center for Promotion of Computational Science and Engineering in JAEA for providing the PC Cluster parallel computer system for the exclusive use of the present calculations.

The authors would like to acknowledge the researchers of the BL04 and BL19 device groups for their cooperation with the neutron intensity measurements.

The authors would also like to acknowledge Dr. K. Haga and Dr. T. Aso in Neutron Source Section of J-PARC, JAEA, for their cooperation to obtain the nuclear heat data.

Finally, the authors would also like to acknowledge the radiation safety group of the MLF for their cooperation with the dose rate measurements.

\section{References}

1) F. Maekawa et al., "First neutron production utilizing J-PARC pulsed neutron source JSNS and neutronic performance dem- 
onstrated," Nucl. Instr. Meth. Phys. Res., A620, 159-165 (2010).

2) Y. Nakahara, T. Tsutsui, NMTC/JAERI A Code System for High Energy Nuclear Reactions and Nucleon-Meson Transport Code, JAERI-M 82-198, Japan Atomic Energy Research Institute (JAERI) (1982), [in Japanese].

3) H. Takada, N. Yoshizawa, N. Kosaka, K. Ishibashi, An Upgrade Version of The Nucleon Meson Transport Code: NMTC/JAERI97, JAERI-Data/Code 98-005, Japan Atomic Energy Research Institute (JAERI) (1998).

4) Y. Nara, N. Otsuka, A. Ohnishi, K. Niita, S. Chiba, "Study of Relativistic Nuclear Collisions at AGS Energies from $\mathrm{p}+\mathrm{Be}$ to $\mathrm{Au}+\mathrm{Au}$ with Hadronic Cascade Model," Phys. Rev., C61, 024901 (1999).

5) H. Nakashima et al., "Research Activities on Neutronics under ASTE Collaboration at AGS/BNL," J. Nucl. Sci. Technol., Suppl. 2, 1155 (2002)

6) F. Maekawa et al., "Analysis of a Neutronic Experiment on a Simulated Mercury Spallation Neutron Target Assembly Bombarded by GeV Protons," Nucl. Sci. Eng., 150, 99 (2005).

7) K. Niita, H. Takada, S. Meigo, Y. Ikeda, "High-energy particle transport code NMTC/JAM," Nucl. Instr. Meth. Phys. Res., B184, 406 (2001).

8) H. Iwase, K. Niita, T. Nakamura, "Development of General-Purpose Particle and Heavy Ion Transport Monte Carlo Code," J. Nucl. Sci. Technol., 39, 1142 (2002).

9) K. Niita, T. Sato, H. Iwase, H. Nose, H. Nakashima, L. Sihver, "PHITS-a particle and heavy ion transport code system," $R a$ diat. Meas., 41, 1080-1090 (2006).

10) T. Nakagawa, K. Shibata, S. Chiba, "Japanese Evaluated Nuclear Data Library version 3 revision-2: JENDL-3.2," J. Nucl. Sci. Technol., 32, 1259 (1995).

11) K. Shibata, T. Fukahori, S. Chiba, N. Yamamuro, "Evaluation of Neutron Nuclear Data for Mercury," J. Nucl. Sci. Technol.,
34, 1171 (1997).

12) K. L. Brown, Ch. Iselin, DECAY TURTLE, CERN 74-02 (1974).

13) T. Kai et al., DCHAIN-SP 2001: High Energy Partcile Induced Radioactivity Calculation Code, JAERI-Data/Code 2001-016. Japan Atomic Energy Research Institute (JAERI) (2001), [in Japanese].

14) D. B. Pelowitz, (ed.), MCNPX User's Manual, Version 2.6.0, LA-CP-07-1473 Los Alamos National Laboratory (LANL) (2008).

15) J. F. Briesmeister (Ed.), MCNP $P^{T M}$ - A General Monte Carlo $N$-Particle Transport Code, Version 4C, LA-13709-M, Los Alamos National Laboratory (LANL) (2000).

16) Home page of Neutron Beam Performance of Material and Life Sciences Experimental Facility in J-PARC home page, http://j-parc.jp/MatLife/en/instrumentation/ns3.html

17) R. E. MacFarlane, Cold-moderator scattering kernel methods, LA-UR-98-655, Los Alamos National Laboratory (LANL) (1998).

18) M. Harada et al., Nuclear heating calculation for JSNS, Proc. ICANS-XVIII, April 25-29, 2007, Dongguan, Guangdong, P R China, 616-626 (2007).

19) S. Meigo et al., " Development of Current Mode Time-of-Flight Technique for Measurement of Thermal Neutron Sprctrum," J. Nucl. Sci. Technol., Suppl. 1, 789-793 (2000).

20) M. Harada et al., "MEASUREMENT OF NEUTRONIC CHARACTERISTICS OF JSNS," Proc. ICANS XIX, PSI-Proceedings 10-01 (2010).

21) T. Aso et al., High Intensity Proton Accelerator Project (J-PARC) Technical Design Report Material \& Life Science Experimental Facility, JAERI-Tech 2004-001, Japan Atomic Energy Research Institute (JAERI), 369-471 (2004). 